\title{
Physics in the Global Monopole Spacetime
}

\author{
E.R. Bezerra de Mello \\ Departamento de Física-CCEN \\ Universidade Federal da Paraíba, C. Postal 5.008, Brazil \\ E-mail: emello@fisica.ufpb.br
}

Received on 2 February, 2001

\begin{abstract}
In this paper we review some important aspects of the global monopole spacetime and present how this manifold modifies, at classical and quantum points of view, the movement of a charged particle. The explicit calculations of the renormalized vacuum expectation values of the energy-momentum tensors, $\left\langle T_{\mu \nu}(x)\right\rangle_{R e n}$., associated with massless bosonic and fermionic fields are also presented. Moreover the effect of the nonzero temperature in this previous formalism is analyzed. Finally, we briefly present other applications of this manifold in the topological inflation and condensed matter system.
\end{abstract}

\section{Introduction}

It is well known that different types of topological objects may have been formed during Universe expansion, such as domain walls, cosmic strings and monopoles[1]. The basic idea is that these topological defects appeared due to breakdown of local or global gauge symmetries. Suppose that we have the Higgs field $\phi^{a}(a=1, \ldots, N)$, whose potential is

$$
V(\phi)=\frac{\lambda}{4}\left(\phi^{2}-\eta_{0}^{2}\right)^{2}, \phi^{2}=\phi^{a} \phi^{a},
$$

where $\eta_{0}$ is the vacuum expectation value and $\lambda$ a coupling constant. This model gives rise to different types of topological defects: Domain wall for $N=1$, cosmic string for $N=2$ and monopoles for $N=3$. The scalar matter field plays the role of an order parameter which outside the defect acquires a nonvanishing value.

In this review we present the field equations for the global monopole in section II, using a specific model coupled to the Einstein equations. In section III, we analyze the classical and quantum motion of a charged particle in the spacetime of an idealized pointlike global monopole, considering the induced electrostatic selfinteraction. In section IV, we calculate the Green functions associate with massless scalar and fermionic fields and obtain the renormalized vacuum expectation values (VEV) of their respective energy-momentum tensors, $\left\langle T_{\mu \nu}(x)\right\rangle_{\text {Ren. }}$. The effect of the temperature in the scalar massless Green function and consequently its influence on the calculation of $\left\langle T_{\mu \nu}\right\rangle$ is evaluated in section V. In section VI, we briefly present other applications of this formalism. Finally we left for section VII our concluding remarks.

\section{Field Equation for a Global Monopole}

\section{A. The Model}

A global monopole is a heavy object formed in the phase transition of a system composed by a selfcoupling iso-scalar triplet $\phi^{a}$, whose original $O(3)$ symmetry is spontaneously broken to $U(1)$ [2].

$$
L=\frac{1}{2} g^{\mu \nu}\left(\partial_{\mu} \phi^{a}\right)\left(\partial_{\nu} \phi^{a}\right)-\frac{\lambda}{4}\left(\phi^{2}-\eta_{0}^{2}\right)^{2} .
$$

The field configuration describing a monopole is

$$
\phi^{a}=\eta_{0} f(r) \hat{x}^{a},
$$

where $x^{a} x^{a}=r^{2}$. The most general static metric tensor with spherical symmetry can be written as

$$
d s^{2}=-B(r) d t^{2}+A(r) d r^{2}+r^{2}\left(d \theta^{2}+\sin ^{2} \phi d \phi^{2}\right) .
$$

The Euler-Lagrange equation for the field $f(r)$ in the metric (4) is:

$$
\frac{\left(r^{2} f^{\prime}\right)^{\prime}}{A r^{2}}+\frac{1}{2 B}\left(\frac{B}{A}\right)^{\prime} f^{\prime}-\frac{2 f}{r^{2}}-\lambda \eta_{0}^{2} f\left(f^{2}-1\right)=0,
$$

where the prime denotes differentiation with respect to $r$. The energy-momentum tensor associated with the matter field is given by

$$
\begin{aligned}
& T_{t}^{t}=-\eta_{0}^{2}\left[\frac{f^{2}}{r^{2}}+\frac{\left(f^{\prime}\right)^{2}}{2 A}+\frac{\lambda}{4} \eta_{0}^{2}\left(f^{2}-1\right)^{2}\right], \\
& T_{r}^{r}=-\eta_{0}^{2}\left[\frac{f^{2}}{r^{2}}-\frac{\left(f^{\prime}\right)^{2}}{2 A}+\frac{\lambda}{4} \eta_{0}^{2}\left(f^{2}-1\right)^{2}\right], \\
& T_{\theta}^{\theta}=T_{\phi}^{\phi}=-\eta_{0}^{2}\left[\frac{\left(f^{\prime}\right)^{2}}{2 A}+\frac{\lambda}{4} \eta_{0}^{2}\left(f^{2}-1\right)^{2}\right] .
\end{aligned}
$$


In flat space the monopole has size $\delta \sim \frac{1}{\sqrt{\lambda} \eta_{0}}$. Its mass is $M \sim \frac{\eta_{0}}{\sqrt{\lambda}}$.

Outside the core $f \sim 1$ and the energy-momentum tensor can be approximated as

$$
T_{t}^{t} \sim T_{r}^{r} \sim-\frac{\eta_{0}^{2}}{r^{2}}, T_{\theta}^{\theta}=T_{\phi}^{\phi} \sim 0
$$

\section{B. Einstein Equations}

Computing the Ricci tensor for the metric tensor (4) one finds [3]:

$$
\begin{aligned}
R_{r r} & =-\frac{B^{\prime \prime}}{2 B}+\frac{B^{\prime}}{4 B}\left(\frac{A^{\prime}}{A}+\frac{B^{\prime}}{B}\right)+\frac{A^{\prime}}{r A}, \\
R_{\theta \theta} & =1-\frac{r}{2 A}\left(\frac{B^{\prime}}{B}-\frac{A^{\prime}}{A}\right)-\frac{1}{A}, \\
R_{t t} & =\frac{B^{\prime \prime}}{2 A}-\frac{B^{\prime}}{4 A}\left(\frac{A^{\prime}}{A}+\frac{B^{\prime}}{B}\right)+\frac{B^{\prime}}{r A} .
\end{aligned}
$$

We also have $R_{\phi \phi}=\sin ^{2} \theta R_{\theta \theta}$.

Combining conveniently these equations we get

$$
\begin{aligned}
\frac{R_{r r}}{2 A}+\frac{R_{t t}}{2 B}+\frac{R_{\theta \theta}}{r^{2}} & =\frac{A^{\prime}}{r A^{2}}+\frac{1}{r^{2}}-\frac{1}{A r^{2}} \\
& =\frac{1}{2}\left(R_{r}^{r}-R_{t}^{t}\right)+R_{\theta}^{\theta},
\end{aligned}
$$

and consequently

$$
(r / A)^{\prime}=1-r^{2}\left[1 / 2\left(R_{r}^{r}-R_{t}^{t}\right)+R_{\theta}^{\theta}\right] .
$$

With the aid of the Einstein equations

$$
R_{\mu \nu}=8 \pi G\left(T_{\mu \nu}-\frac{1}{2} g_{\mu \nu} T\right)
$$

we obtain

$$
(r / A)^{\prime}=1+8 \pi G r^{2} T_{t}^{t} .
$$

To find the other component of the metric tensor we write

$$
\begin{aligned}
\frac{R_{r r}}{A}+\frac{R_{t t}}{B} & =\frac{1}{r A}\left(\frac{A^{\prime}}{A}+\frac{B^{\prime}}{B}\right) \\
& =R_{r}^{r}-R_{t}^{t} .
\end{aligned}
$$

Integrating with the condition

$$
A(r) B(r)_{\mid r=\infty}=1
$$

we obtain

$$
B(r)=\frac{1}{A(r)} \exp \left[8 \pi G \int_{\infty}^{r}\left(T_{r}^{r}-T_{t}^{t}\right) r A(r) d r\right] .
$$

The general solution of (12) is

$$
A(r)^{-1}=1-8 \pi G \eta_{0}^{2}-2 G M(r) / r
$$

where $M(r)$ is given by

$M(r)=4 \pi \eta_{0}^{2} \int_{0}^{r}\left[\frac{\left(f^{\prime}\right)^{2}}{2 A}+\frac{f^{2}-1}{r^{2}}+\frac{\lambda \eta_{0}^{2}}{4}\left(f^{2}-1\right)\right] r^{2} d r$.

Because $T_{t}^{t} \sim T_{r}^{r}$ outside the core of the monopole, in this region we have

$$
B(r)=1 / A(r)
$$

Also we can analyze the behavior of the function $f$. Introducing news variables defined as[4]

$$
x=\sqrt{\lambda} \eta_{0} r, \Lambda=8 \pi G \eta_{0}^{2},
$$

one finds the asymptotic expansion

$$
f(x)=1-\frac{1}{x^{2}}-\frac{3 / 2-\Lambda}{x^{4}}+O\left(1 / x^{6}\right) .
$$

Now let us go back to the metric tensor. Neglecting the mass term and rescaling the $t$ variable we can rewrite the monopole metric as

$$
d s^{2}=-d t^{2}+\frac{d r^{2}}{\alpha^{2}}+r^{2} d \Omega^{2}
$$

where $\alpha^{2}=1-8 \pi G \eta_{0}^{2}=1-\Lambda<1$. The above metric tensor describes an idealized pointlike global monople (G-M) defect.

The spacetime described by (20) has the following interesting features:

i) It is not flat: the scalar curvature $R=R_{\mu}^{\mu}=2 \frac{\left(1-\alpha^{2}\right)}{r^{2}}$.

ii) There is no Newtonian potential: $g_{t t}=-1$.

iii) The surface $\theta=\pi / 2$ has geometry of a cone, with deficit angle $\delta \Omega=8 \pi^{2} G \eta_{0}^{2}$.

iv) The solid angle of a sphere of unit radius is $4 \pi^{2} \alpha^{2}$, so smaller than $4 \pi^{2}$. There is a solid angle deficit $\delta \Omega=32 \pi^{2} G \eta_{0}^{2}$.

v) Because $T_{00} \sim \eta^{2} / r^{2}$, outside the global monopole the total energy is linearly divergent at large distance: $E(r) \sim 4 \pi G \eta_{0}^{2} r$.

Now after this brief review about the global monopole spacetime, let us analyze some classical and quantum effects produced by this geometry on the movement of a charged particle.

\section{Classical Effects}

It is well known that a charged/massive particle when placed in the spacetime of a cosmic string becomes subjected to a repulsive/attractive self-interaction[5]. A similar phenomenon also occur in the spacetime produced by a global monopole. These self-interactions are given by[6]:

$$
U=\frac{K}{r}
$$


where $r$ is the distance from the particle to the monopole and

$$
K=q^{2} S(\alpha) / 2>0
$$

for the electrostatic case, or

$$
K=-G m^{2} S(\alpha) / 2<0 \text {, }
$$

for the induced gravitational one. The numerical factor $S(\alpha)$ is a function of the parameter $\alpha$

$$
S(\alpha)=\sum_{l=0}^{\infty}\left[\frac{2 l+1}{\sqrt{\alpha^{2}+4 l(l+1)}}-1\right],
$$

which is finite and positive for $\alpha<1$.

These induced self-interactions are consequence of the distortion on the particle's field caused by the curvature and/or the non-trivial topology of the spacetime.

Briefly speaking these effects can be explained by calculating the Newtonian gravitational self-interaction of an arbitrary mass distribution outside the monopole core

$$
U_{G}=-\frac{1}{2} G \iint d \mathbf{r} \mathbf{d} \mathbf{r}^{\prime} \rho_{\mathbf{m}}(\mathbf{r}) \mathbf{G}\left(\mathbf{r}, \mathbf{r}^{\prime}\right) \rho_{\mathbf{m}}\left(\mathbf{r}^{\prime}\right),
$$

where $\rho_{m}$ is the mass density. Similarly, for the electrostatic self-interaction of an arbitrary charge distribution we have

$$
U_{E}=\frac{1}{2} G \iint d \mathbf{r} \mathbf{d} \mathbf{r}^{\prime} \rho_{\mathbf{q}}(\mathbf{r}) \mathbf{G}\left(\mathbf{r}, \mathbf{r}^{\prime}\right) \rho_{\mathbf{q}}\left(\mathbf{r}^{\prime}\right),
$$

where now $\rho_{q}$ is the charge density.

For an isolated particle at some specific position $r$, we get

$$
U_{G}=-\frac{1}{2} G m^{2} G_{R}(\mathbf{r}, \mathbf{r})
$$

and

$$
U_{E}=\frac{1}{2} q^{2} G_{R}(\mathbf{r}, \mathbf{r}),
$$

where $G_{R}\left(\mathbf{r}, \mathbf{r}^{\prime}\right)$ is the renormalized Green function defined as

$$
G_{R}\left(\mathbf{r}, \mathbf{r}^{\prime}\right)=G_{\alpha}\left(\mathbf{r}, \mathbf{r}^{\prime}\right)-G_{H}\left(\mathbf{r}, \mathbf{r}^{\prime}\right),
$$

being $G_{\alpha}\left(\mathbf{r}, \mathbf{r}^{\prime}\right)$ the Green function defined in the monopole space section:

$$
G_{(\alpha)}\left(\mathbf{r}, \mathbf{r}^{\prime}\right)=\frac{1}{\alpha r_{>}} \sum_{l=0}^{\infty} \frac{2 l+1}{2 \lambda_{l}+1}\left(\frac{r_{<}}{r_{>}}\right)^{\lambda_{l}} P_{l}(\cos \gamma),
$$

where $\lambda_{l}=-\frac{1}{2}+\frac{\sqrt{\alpha^{2}+4 l(l+1)}}{2 \alpha}$.

The Hadamard function, $G_{H}\left(\mathbf{r}, \mathbf{r}^{\prime}\right)$, associated with the three-dimensional Laplace-Beltrami operator is

$$
G_{H}\left(\mathbf{r}, \mathbf{r}^{\prime}\right)=\frac{1}{2 \sigma\left(\mathbf{r}, \mathbf{r}^{\prime}\right)}
$$

being $\sigma\left(\mathbf{r}, \mathbf{r}^{\prime}\right)$ the one-half of the geodesic distance between the two points $\mathbf{r}$ and $\mathbf{r}^{\prime}$ is the space section of (20). Because we are interested to evaluate the renormalized Green function in the coincidence limit, let us take first $\Omega=\Omega^{\prime}$. For this case the three-dimensional line element in the G-M spacetime is

$$
d \vec{l}^{2}=\frac{d r^{2}}{\alpha^{2}}
$$

which give us

$$
2 \sigma\left(\mathbf{r}, \mathbf{r}^{\prime}\right)=\frac{\left|r-r^{\prime}\right|}{\alpha} .
$$

So after some intermediate calculations we get

$$
G_{R}(\mathbf{r}, \mathbf{r})=\frac{1}{r} S(\alpha)
$$

which is finite for $r>0$.

The presence of this induced electrostatic selfinteraction is relevant in the analysis of the movement of an electric charged particle placed in the spacetime of a global monopole. In order to do that we take into account the self-interaction as the zeroth component of the four-vector $A_{\mu}$. The next subsection is devoted to this investigation.

\section{A. Classical Analysis of the Motion}

For the relativistic classical analysis of this movement we shall use the Hamilton-Jacobi (H-J) formalism. According this formalism, our first set of equation is given by[7]

$$
M \frac{d x^{\mu}}{d \lambda}+q A^{\mu}=g^{\mu \nu} \frac{\partial S}{\partial x^{\nu}},
$$

$\lambda$ being a parameter along the classical trajectory of the particle.

The second set of equation is given by

$$
g^{\mu \nu}\left(\frac{\partial S}{\partial x^{\mu}}-q A_{\mu}\right)\left(\frac{\partial S}{\partial x^{\nu}}-q A_{\nu}\right)=-M^{2},
$$

where $S$ is the functional given by

$$
S=-E t+R(r)+\Theta(\theta)+L_{Z} \phi,
$$

with $R(r)$ and $\Theta(\theta)$ being unknown functions and $E$ and $L_{Z}$ constants of the movement.

Integrating (32) and (33) we can obtain the equations of the trajectories for different situations as shown below. Defining $u=\frac{1}{r}$, we get, for the surface $\theta=\pi / 2$, the following solutions:

$$
\begin{aligned}
u(\phi)=\frac{E K}{L^{2}-K^{2}} & +\frac{\sqrt{\left(E^{2}-M^{2}\right) L^{2}+M^{2} K^{2}}}{L^{2}-K^{2}} \\
& \times \cos \left[\frac{\sqrt{L^{2}-K^{2}}}{L_{Z}} \alpha\left(\phi-\phi_{0}\right)\right]
\end{aligned}
$$


for $L^{2}>K^{2}$,

$$
\begin{aligned}
u(\phi)=\frac{E K}{K^{2}-L^{2}} & +\frac{\sqrt{\left(E^{2}-M^{2}\right) L^{2}+M^{2} K^{2}}}{K^{2}-L^{2}} \\
& \times \cosh \left[\frac{\sqrt{K^{2}-L^{2}}}{L_{Z}} \alpha\left(\phi-\phi_{0}\right)\right],
\end{aligned}
$$

for $L^{2}<K^{2}$ and

$$
u(\phi)=\frac{M^{2}}{2 E K}-\frac{E^{2} \alpha^{2} \chi^{2}\left(\phi-\phi_{0}\right)^{2}}{2 K},
$$

for $L^{2}=K^{2}$.

From the above equations is possible to see that the first set of solutions presents bounded trajectory for $E^{2}<M^{2}$ and $K<0$, which happens for the unphysical case when $\alpha>1$.

\section{B. Quantum Analysis of the Motion}

For the analysis of the quantum motion we should use the relativistic Klein-Gordon equation or Dirac one if the particle is a boson or fermion, respectively.

\section{Bosonic Case}

For the spin 0 case the Klein-Gordon equation written in a covariant form reads

$$
\begin{gathered}
{\left[\square-\frac{i q A^{\mu}}{\sqrt{-g}}\left(\partial_{\mu} \sqrt{-g}\right)-i q\left(\partial_{\mu} A^{\mu}\right)-2 i q A^{\mu} \partial_{\mu}\right.} \\
\left.-q^{2} A^{\mu} A_{\mu}-\xi R(x)-M^{2}\right] \Phi(x)=0,
\end{gathered}
$$

with

$$
\square \Phi(x)=\frac{1}{\sqrt{-g}} \partial_{\mu}\left[\sqrt{-g} g^{\mu \nu} \partial_{\nu} \Phi(x)\right] .
$$

where $g=\operatorname{det}\left(g_{\mu \nu}\right)$. In the above equation we also considered the non-minimal coupling between the scalar field $\Phi$ with the scalar curvature $R$ of the manifold.

Now applying this formalism for the G-M spacetime we have

$$
\begin{gathered}
{\left[-\partial_{t^{2}}+\frac{\alpha^{2}}{r^{2}} \partial_{r}\left(r^{2} \partial_{r}\right)-\frac{\vec{L}^{2}}{r^{2}}-2 i \frac{K}{r} \partial_{t}+\frac{K^{2}}{r^{2}}\right.} \\
\left.-M^{2}-\xi \frac{\eta}{r^{2}}\right] \Phi(x)=0 .
\end{gathered}
$$

Because our metric tensor is static and the self-potential is time-independent, we can adopt for the wave function the form

$$
\Phi(x)=C \exp (-i E t) R(r) Y_{l, m}(\theta, \phi)
$$

where $E$ is the particle's self-energy. The solution for the radial differential equation are expressed in terms of hypergeometric functions as

$$
R(r)=r^{\lambda_{l}} e^{i k r} M\left(\lambda_{l}+1+i \beta, 2\left(\lambda_{l}+1\right) ;-2 i k r\right),
$$

where the effective angular quantum number is

$$
\lambda_{l}=-\frac{1}{2}+\frac{\sqrt{\alpha^{2}+4\left[l(l+1)+\xi \eta-K^{2}\right]}}{2 \alpha}
$$

with $\eta=2\left(1-\alpha^{2}\right)=2 \Lambda$ and

$$
k=\frac{\sqrt{E^{2}-M^{2}}}{\alpha}, \beta=\frac{E K}{\alpha^{2} k} .
$$

From the radial solution is possible to obtain the phase shift $\delta_{l}$, which is the most relevant parameter in the calculation of the scattering amplitude.

If we are inclined to consider the possibility of this system to present bound states, we have to assume that the parameter $\alpha>1$, in which case $K<0$. So, taking $E^{2}<M^{2}$, we get discrete values for the self-energy given by

$$
E_{n, l}=M\left[\frac{\alpha^{2}\left(n+\lambda_{l}+1\right)^{2}}{K^{2}+\alpha^{2}\left(n+\lambda_{l}+1\right)}\right]^{1 / 2},
$$

with $n=0,1,2 \ldots$

\section{Fermionic Case}

Considering now a fermionic particle, we must take the Dirac equation in the covariant form

$$
\left[i \gamma^{\mu}(x)\left(\partial_{\mu}+i q A_{\mu}-\Gamma_{\mu}(x)\right)-M\right] \Psi(x)=0,
$$

where $\Gamma_{\mu}(x)$ is the spin coefficient. Using an appropriated tetrad basis for the G-M spacetime we get[7]:

$$
\begin{gathered}
{\left[i \gamma^{(0)} \partial_{t}+i \alpha \gamma^{(r)} \partial_{r}+\frac{i}{r} \gamma^{(\theta)} \partial_{\theta}+\frac{i}{r \sin \theta} \gamma^{\phi} \partial_{\phi}\right.} \\
\left.+i \frac{(\alpha-1)}{r} \gamma^{(r)}-q \gamma^{(0)} A_{0}-M\right] \Psi(x)=0
\end{gathered}
$$

Also using the standard procedure it is possible to obtain the set of solution for the equation above. They are expressed in terms of hypergeometric functions. Again from the solution we can calculate the phase shift, $\delta_{j}$. If again we admit the unphysical situation where $\alpha>1$, it is possible to find bound states and the explicit expression for the discrete self-energy

$$
E_{n, j}=M\left[1+\frac{K^{2}}{\left(n \alpha+\sqrt{(j+1 / 2)^{2}-K^{2}}\right)^{2}}\right]^{-1 / 2} .
$$




\section{Quantum Effects}

The non-trivial topology of the G-M spacetime implies that the vacuum expectation value (VEV) of the renormalized energy-momentum tensor associated with an arbitrary collection of conformal massless quantum field must be different from zero[8].

The explicit calculations for $\left\langle T_{\mu \nu}(x)\right\rangle_{\text {Ren }}$ for a massless scalar field[9] and fermionic one[12] have been developed. Also, recently thermal effects have been considered in this context[13].

Below we present the main effects due to this spacetime in the VEV of some quantum operators.

\section{A. Scalar Case}

The Euclidean version of the G-M metric tensor is given by

$$
d s^{2}=d \tau^{2}+\frac{d r^{2}}{\alpha^{2}}+r^{2} d \Omega^{2} .
$$

The massless scalar field propagator obeys the differential equation below

$$
(-\square+\xi R) G_{E}\left(x, x^{\prime}\right)=\frac{\delta^{4}\left(x, x^{\prime}\right)}{\sqrt{g}},
$$

where we have included the non-minimal coupling between the propagator with the geometry. The Eu- clidean Green function can be evaluated using the heat kernel approach:

$$
\begin{aligned}
G_{E}\left(x, x^{\prime}\right) & =\int_{0}^{\infty} d s \exp \{-s(-\square+\xi R)\} \frac{\delta^{4}\left(x, x^{\prime}\right)}{\sqrt{g}} \\
& =\int_{0}^{\infty} d s K\left(x, x^{\prime} ; s\right)
\end{aligned}
$$

In order to obtain the heat kernel, $K\left(x, x^{\prime} ; s\right)$, let us solve the eigenfunction equation below

$$
(-\square+\xi R) \Phi_{\lambda}(x)=\lambda^{2} \Phi_{\lambda}(x) .
$$

The complete result for the equation above is:

$$
\Phi_{\lambda}(x)=\sqrt{\frac{\alpha p}{2 \pi r}} \exp (-i \omega t) J_{\nu_{l}}(p r) Y_{l, m}(\theta, \phi)
$$

with eigenvalues $\lambda^{2}=\omega^{2}+p^{2} \alpha^{2} \geq 0$ and $\nu_{l}=$ $\alpha^{-1} \sqrt{(l+1 / 2)^{2}+2\left(1-\alpha^{2}\right)(\xi-1 / 8)}$. These solutions obey the completeness relation

$$
\sum_{\lambda} \Phi_{\lambda}(x) \Phi_{\lambda}^{*}(x)=\frac{\delta^{4}\left(x, x^{\prime}\right)}{\sqrt{g}} .
$$

Now we are in position to obtain the heat kernel by

$$
\begin{aligned}
K\left(x, x^{\prime} ; s\right) & =\int_{-\infty}^{\infty} d \omega \int_{0}^{\infty} d p \sum_{l, m} \Phi_{\lambda}(x) \Phi_{\lambda}\left(x^{\prime}\right) \exp \left(-s \lambda^{2}\right) \\
& =\frac{\exp \left(-\frac{\Delta \tau^{2} \alpha^{2}+r^{2}+r^{\prime 2}}{4 \alpha^{2} s}\right)}{16(\pi s)^{3 / 2}\left(r r^{\prime}\right)^{1 / 2}} \\
& \times \sum_{l=0}^{\infty}(2 l+1) I_{\nu_{l}}\left(\frac{r r^{\prime}}{2 \alpha^{2} s}\right) P_{l}(\cos \gamma) .
\end{aligned}
$$

We can easily verify that for $\alpha=1, \nu_{l}=l+1 / 2$ and in this case it is possible to get a closed expression for the heat kernel [14]:

$$
K\left(x, x^{\prime} ; s\right)=\frac{1}{16 \pi^{2} s^{2}} \exp \left(-\frac{\left(x-x^{\prime}\right)^{2}}{4 s}\right) .
$$

Finally we can obtain the Euclidean Green function by integrating (53). Our result is

$$
\begin{aligned}
G\left(x, x^{\prime}\right) & =\frac{1}{8 \pi^{2} r r^{\prime}} \sum_{l=0}^{\infty}(2 l+1) \\
& \times Q_{\nu_{l}-1 / 2}\left(\frac{\alpha^{2} \Delta \tau^{2}+r^{2}+r^{\prime 2}}{2 r r^{\prime}}\right) P_{l}(\cos \gamma) .
\end{aligned}
$$

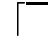

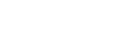


where $G_{H}\left(x, x^{\prime}\right)$ is the Hadamard function given by[9]

$$
\begin{aligned}
G_{H}\left(x, x^{\prime}\right) & =\frac{1}{16 \pi^{2}}\left[\frac{2}{\sigma\left(x, x^{\prime}\right)}\right. \\
& \left.+(\xi-1 / 6) R(x) \ln \left(\frac{\mu^{2} \sigma\left(x, x^{\prime}\right)}{2}\right)\right]
\end{aligned}
$$

where $\mu$ is an arbitrary scale introduced in order to avoid the infrared divergence and $\sigma\left(x, x^{\prime}\right)$ the one-half of the square of the geodesic distance between the points $x$ and $x^{\prime}$. Because we are interested in the coincidence limit, let us set first $\Omega^{\prime}=\Omega$ in (57). For this case we get $\sigma\left(x, x^{\prime}\right)=\frac{\left(r-r^{\prime}\right)^{2}}{2 \alpha^{2}}$. Our above expressions reduce themselves to

$$
G\left(r, r^{\prime}\right)=\frac{1}{8 \pi^{2} r r^{\prime}} \sum_{l=0}^{\infty}(2 l+1) Q_{\nu_{l}-1 / 2}\left(\frac{r^{2}+r^{\prime 2}}{2 r r^{\prime}}\right)
$$

and

$$
\begin{aligned}
G_{H}\left(r, r^{\prime}\right) & =\frac{1}{16 \pi^{2}}\left[\frac{4 \alpha^{2}}{\left(r-r^{\prime}\right)^{2}}\right. \\
& \left.+\frac{2 \eta}{r^{2}}(\xi-1 / 6) \ln \left(\frac{\mu^{2}\left(r-r^{\prime}\right)^{2}}{4 \alpha^{2}}\right)\right]
\end{aligned}
$$

Because the dependence of $\nu_{l}$ with $l$ is not simple one, it is not possible to develop the summation in the angular quantum number $l$ in (59) in an exact way; however if we remember that the parameter $\alpha^{2}=1-\Lambda$ is close to the unity for realistic models ${ }^{1}$, it is possible to expand $\nu_{l}$ in powers of $\Lambda$ and consequently to obtain an approximate expression for (59). So in lowest order in $\Lambda$ and using appropriate integral representation for the Legendre function we get:

$$
\begin{aligned}
G\left(r, r^{\prime}\right) & =\frac{1}{8 \sqrt{2} \pi^{2} r r^{\prime}} \int_{\rho}^{\infty} d t \frac{1}{\sqrt{\cosh t-\cosh \rho}} \\
& \times \sum_{l=0}^{\infty}(2 l+1) e^{-\nu_{l} t}
\end{aligned}
$$

with $\cosh \rho=\left(r+r^{\prime}\right)^{2} / 2 r r^{\prime}$. Moreover evaluating the summation in $l$ up to the first order in $\Lambda$, we have:

$$
\begin{aligned}
S(t) & =\sum_{l=0}^{\infty}(2 l+1) e^{-\nu_{l} t} \\
& =e^{-t / 2} \frac{1+e^{-t}}{\left(1-e^{-t}\right)^{2}}\left[1-\frac{2 \Lambda t}{1-e^{-2 t}}\right. \\
& {\left.\left[\xi\left(1-e^{-t}\right)^{2}+e^{-t}\right]\right] . }
\end{aligned}
$$

Also the logarithmic term in the Hadamard function can be expressed in term of $Q_{0}(\cosh \rho)=\ln \left(\frac{r+r^{\prime}}{r-r^{\prime}}\right)$ and consequently in integral form. So taking into account all these considerations we found, up to the first order in $\Lambda$

$$
\left\langle\Phi^{2}(x)\right\rangle_{\text {Ren }}=\lim _{r^{\prime} \rightarrow r}\left[G\left(r, r^{\prime}\right)-G_{H}\left(r, r^{\prime}\right)\right]
$$

$$
=-\Lambda \frac{(p-2 \xi q)}{8 \sqrt{2} \pi^{2} r^{2}}-\Lambda \frac{(\xi-1 / 6)}{4 \pi^{2} r^{2}} \ln (\mu r)
$$

where $p$ and $q$ are two numbers given by the integrals below

$$
\begin{aligned}
p= & \int_{0}^{\infty} d t \frac{e^{-t / 2}}{\sqrt{\cosh t-1}}\left[\frac{1}{3}+\left[\frac{t}{\sinh t}-1\right]\right. \\
& \left.\times \frac{1+e^{-t}}{\left(1-e^{-t}\right)^{2}}\right]
\end{aligned}
$$

and

$$
q=\int_{0}^{\infty} d t \frac{e^{-t / 2}}{\sqrt{\cosh t-1}}\left[1-\frac{t\left(1+e^{-t}\right)}{1-e^{-2 t}}\right] .
$$

These two integrals are finite and can be evaluated numerically. The results are $p=-0.39$ and $q=-1.41[9]$.

\section{Evaluation of $\left\langle T_{\mu}^{\nu}(x)\right\rangle_{\text {Ren }}$}

In Ref. [9] the general structure of the renormalized VEV of the energy-momentum tensor for a massless scalar field in the G-M spacetime is presented. There, this expression was obtained on basis of dimensional arguments, symmetries, trace anomaly and the explicit calculation of the VEV of the square of the field operator. Their conclusion is that the VEV for this energymomentum tensor has the following structure:

$$
\left\langle T_{\mu}^{\nu}(x)\right\rangle_{R e n}=\frac{1}{16 \pi^{2} r^{4}}\left[A_{\mu}^{\nu}(\xi, \Lambda)+B_{\mu}^{\nu}(\xi, \Lambda) \ln (\mu r)\right]
$$

where $B_{\mu}^{\nu}$ and $A_{\mu}^{\nu}$ are diagonal tensors The explicit expression for the latter is

$$
\begin{aligned}
A_{\mu}^{\nu} & =\operatorname{diag}\left(T+A_{r}^{r}-B_{r}^{r}, A_{r}^{r},-A_{r}^{r}+1 / 2 B_{r}^{r},\right. \\
& \left.-A_{r}^{r}+1 / 2 B_{r}^{r}\right) .
\end{aligned}
$$

For the particular value $\xi=1 / 6, T$ is given by the trace anomaly. We have $\left\langle T_{\mu}^{\mu}(x)\right\rangle_{\text {Ren }}=T / 16 \pi^{2} r^{4}=$ $\Lambda / 770 \pi^{2} r^{4}(1-\Lambda / 2)$.

\section{B. Fermionic Case}

The spinor Feynman propagator is defined as follows[10]

$$
i S_{F}\left(x, x^{\prime}\right)=\left\langle T\left(\psi(x) \bar{\psi}\left(x^{\prime}\right)\right)\right\rangle
$$

This propagator obeys the following differential equation

$$
(i \not \nabla-M) S_{F}\left(x, x^{\prime}\right)=\frac{1}{\sqrt{-g}} \delta^{4}\left(x, x^{\prime}\right) I_{(4)},
$$

\footnotetext{
${ }^{1}$ In fact for a typical grand unified theory the parameter $\eta_{0}$ is of order $10^{16} \mathrm{Gev}$. So $1-\alpha^{2}=\Lambda \sim 10^{-5}$
} 
where $g=\operatorname{det}\left(g_{\mu \nu}\right)$. The spinor covariant derivative is given by

$$
\not=e_{(a)}^{\mu}(x) \gamma^{(a)}\left(\partial_{\mu}+\Gamma_{\mu}\right),
$$

with the spin connection $\Gamma_{\mu}$ given in terms of the $\gamma$ matrix and the basis tetrad $e_{(a)}^{\mu}$ in the usual way:

$$
\Gamma_{\mu}=-\frac{1}{4} \gamma^{(a)} \gamma^{(b)} e_{(a)}^{\nu} e_{(b) \nu ; \mu}
$$

If a bispinor $D_{F}\left(x, x^{\prime}\right)$ satisfies the differential equation

$$
\left(\square-M^{2}-\frac{1}{4} R(x)\right) D_{F}\left(x, x^{\prime}\right)=-\frac{1}{\sqrt{-g}} \delta^{(4)}\left(x, x^{\prime}\right),
$$

where the generalized d'Alembertian operator is expressed by

$$
\square=g^{\mu \nu} \nabla_{\mu} \nabla_{\nu}=g^{\mu \nu}\left(\partial_{\mu} \nabla_{\nu}+\Gamma_{\mu} \nabla_{\nu}-\Gamma_{\mu \nu}^{\alpha} \nabla_{\alpha}\right),
$$

then the spinor Feynman propagator may be written as

$$
S_{F}\left(x, x^{\prime}\right)=(i \not \nabla+M) D_{F}\left(x, x^{\prime}\right) .
$$

Now after this brief review, let us specialize in the calculation of the spin Feynman propagator in the G-M spacetime.

Using appropriate basis tetrad for this spacetime, the only non-vanishing spin connections are[12]:

$$
\Gamma_{2}=\frac{1-\alpha}{2}\left[\gamma^{(1)} \gamma^{(2)} \cos \phi+\gamma^{(2)} \gamma^{(3)} \sin \phi\right]
$$

and

$$
\begin{aligned}
\Gamma_{3} & =-\frac{1-\alpha}{2}\left[\gamma^{(1)} \gamma^{(2)} \sin \theta+\gamma^{(1)} \gamma^{(3)} \sin \phi \cos \theta\right. \\
& \left.-\gamma^{(2)} \gamma^{(3)} \cos \theta \cos \phi\right] \sin \theta
\end{aligned}
$$

We also adopt the following representation for the $\gamma$ matrix

$$
\gamma^{(0)}=\left(\begin{array}{cc}
1 & 0 \\
0 & -1
\end{array}\right), \gamma^{(k)}=\left(\begin{array}{cc}
0 & \sigma^{k} \\
-\sigma^{k} & 0
\end{array}\right) .
$$

These matrices obey the anticommutator relation $\left\{\gamma^{(a)}, \gamma^{(b)}\right\}=-2 \eta^{(a)(b)}$.

After some intermediate steps we get $^{2}$

$$
\begin{aligned}
\square & =-\frac{1}{\alpha^{2}} \partial_{t}^{2}+\frac{\alpha^{2}}{r^{2}} \partial_{r}\left(r^{2} \partial_{r}\right)-\frac{\vec{L}^{2}}{r^{2}} \\
& -\frac{(1-\alpha)^{2}}{2 r^{2}}-\frac{1-\alpha}{r^{2}} \vec{\Sigma} \cdot \vec{L},
\end{aligned}
$$

where

$$
\vec{\Sigma}=\left(\begin{array}{cc}
\vec{\sigma} & 0 \\
0 & \vec{\sigma}
\end{array}\right)
$$

The system that we shall consider consists of a massless left-handed fermionic field. For this case the Dirac equation reduces to a $2 \times 2$ matrix differential equation as shown below:

$$
i \mathcal{P}_{L} \chi=0
$$

where

$$
\begin{aligned}
\not{D}_{L} & =i\left[\frac{1}{\alpha} \partial_{t}-\alpha \sigma^{(r)} \partial_{r}-\frac{1}{r} \sigma^{(\theta)} \partial_{\theta}\right. \\
& \left.-\frac{1}{r \sin \theta} \sigma^{(\phi)} \partial_{\phi}+\frac{1-\alpha}{r} \sigma^{(r)}\right],
\end{aligned}
$$

with $\sigma^{(u)}=\vec{\sigma} \cdot \hat{u}, \hat{u}$ denoting the usual unit vector along the three spatial directions in spherical coordinates.

The Feynman two-component propagator obeys now the equation

$$
i \not_{l} S_{F}\left(x, x^{\prime}\right)=\frac{1}{\sqrt{-g}} \delta^{(4)}\left(x, x^{\prime}\right) I_{(2)},
$$

and can be given in terms of the bispinor $G_{F}\left(x, x^{\prime}\right)$ by

$$
S_{F}\left(x, x^{\prime}\right)=i \not_{L} G_{F}\left(x, x^{\prime}\right),
$$

where now this bispinor obeys the $2 \times 2$ differential equation below:

$$
\hat{K} G_{F}\left(x, x^{\prime}\right)=-\frac{1}{\sqrt{-g}} \delta^{(4)}\left(x, x^{\prime}\right) I_{(2)},
$$

with

$$
\hat{K}=-\frac{1}{\alpha^{2}} \partial_{t}^{2}+\frac{\alpha^{2}}{r^{2}} \partial_{r} r^{2} \partial_{r}-\frac{\vec{L}^{2}}{r^{2}}-\frac{1-\alpha}{r^{2}}(1+\vec{\sigma} \cdot \vec{L}) .
$$

The VEV of the energy-momentum tensor can be expressed in terms of the Euclidean Green function $G_{E}\left(x, x^{\prime}\right)=-i G_{F}\left(x, x^{\prime}\right)$. Now we shall calculate $G_{E}\left(x, x^{\prime}\right)$ by using the solution of the eigenvalue equation below

$$
\hat{K} \Phi_{\lambda}(x)=-\lambda^{2} \Phi_{\lambda}(x),
$$

with $\lambda^{2} \geq 0$, so we can write

$$
G_{E}\left(x, x^{\prime}\right)=\sum_{\lambda} \frac{\Phi_{\lambda}(x) \Phi_{\lambda}^{+}\left(x^{\prime}\right)}{\lambda} .
$$

The normalized eigenfunction can be written as

$$
\Phi_{\lambda}^{(k)}(x)=\sqrt{\frac{p}{2 \pi r}} e^{-i E \tau} J_{\nu_{k}}(p r) \phi_{j, m_{j}}^{(k)}, k=1,2,
$$

with

$$
\lambda^{2}=\frac{E^{2}}{\alpha^{2}}+\alpha^{2} p^{2},
$$

and

$$
\nu_{1}=\frac{l+1}{\alpha}-\frac{1}{2}, \nu_{2}=\frac{l}{\alpha}+\frac{1}{2} .
$$

In the above equation $\phi_{j, m_{j}}^{(k)}$ are the spinor spherical harmonic eigenfunctions of the operators $\vec{L}^{2}$ and $\sigma \cdot \vec{L}[11]$.

\footnotetext{
${ }^{2}$ Here in this section we are using the following metric tensor: $g_{\mu \nu}=\operatorname{diag}\left(-\alpha^{2}, 1 / \alpha^{2}, r^{2}, r^{2} \sin ^{2}(\theta)\right)$.
} 
The expression for $G_{E}\left(x, x^{\prime}\right)$ can be given by

$$
\begin{aligned}
G_{E}\left(x, x^{\prime}\right) & =\int_{-\infty}^{\infty} d E \int_{0}^{\infty} d p \\
& \times \sum_{j, m_{j}} \frac{\Phi_{\lambda}^{(1)}(x) \phi_{\lambda}^{(1)+}\left(x^{\prime}\right)+\Phi_{\lambda}^{(2)}(x) \phi_{\lambda}^{(2)+}\left(x^{\prime}\right)}{E^{2} / \alpha^{2}+\alpha^{2} p^{2}} .
\end{aligned}
$$

Substituting $\Phi_{\lambda}^{(k)}(x)$ in the equation above we get

$$
\begin{gathered}
G_{E}\left(x, x^{\prime}\right)=\frac{1}{2 \pi r r^{\prime}} \sum_{j, m_{j}}\left[Q_{\nu_{1}-1 / 2}(u) C_{j, m_{j}}^{(1)}\left(\Omega, \Omega^{\prime}\right)\right. \\
\left.+Q_{\nu_{2}-1 / 2}(u) C_{j, m_{j}}^{(2)}\left(\Omega, \Omega^{\prime}\right)\right],
\end{gathered}
$$

where $Q_{\nu}(z)$ is the Legendre function, $u=1+\left(\alpha^{4} \Delta \tau^{2}+\right.$ $\left.\Delta r^{2}\right) / 2 r r^{\prime}$ and $C_{j, m_{j}}^{(k)}\left(\Omega, \Omega^{\prime}\right)=\phi_{j, m_{j}}^{(k)}(\Omega) \phi_{j, m_{j}}^{(k)+}\left(\Omega^{\prime}\right)$.

Again we can see that for $\alpha=1, \nu_{1}=\nu_{2}=l+1 / 2$ and we get a closed form for $G_{E}\left(x, x^{\prime}\right)$

$$
G_{E}\left(x, x^{\prime}\right)=\frac{1}{8 \pi^{2}} \frac{1}{\sigma\left(x, x^{\prime}\right)} I_{(2)},
$$

where $2 \sigma\left(x, x^{\prime}\right)=\Delta \tau^{2}+\left(\vec{r}-\vec{r}^{\prime}\right)^{2}$.

Now we can express the spinor Green function as

$$
\begin{aligned}
& S_{F}\left(x, x^{\prime}\right)=i\left[\frac{1}{\alpha} \partial_{t}-\alpha \sigma^{(r)} \partial_{r}+\frac{1}{r} \sigma^{(r)} \vec{\sigma} \cdot \vec{L}\right. \\
& \left.+\frac{1-\alpha}{r} \sigma^{(r)}\right] G_{E}(x, x) .
\end{aligned}
$$

\section{Vacuum Expectation Value}

Also, as in the scalar case, the general structure of the VEV of the energy-momentum tensor associated with a massless fermionic field in the G-M spacetime has the following structure:

$$
\left\langle T_{\mu}^{\nu}(x)\right\rangle_{R e n}=\frac{1}{8 \pi^{2} r^{4}}\left[A_{\mu}^{\nu}+B_{\mu}^{\nu} \ln \left(\frac{\mu r}{\alpha}\right)\right],
$$

where

$$
\begin{aligned}
& A_{\mu}^{\nu}=\operatorname{diag}\left(A_{0}^{0},-T+A_{0}^{0}+B_{0}^{0},\right. \\
& \left.\quad T-A_{0}^{0}-1 / 2 B_{0}^{0}, T-A_{0}^{0}-1 / 2 B_{0}^{0}\right)
\end{aligned}
$$

and

$$
B_{\mu}^{\nu}=B_{0}^{0} \operatorname{diag}(1,1,-1,-1) .
$$

Because for this system $T:=\frac{r^{4} T r a_{2}}{2}=-\frac{1-\alpha^{4}}{60}$, our problem of finding (94) consists to obtain the two components $A_{0}^{0}$ and $B_{0}^{0}$ only.

Using the point-splitting approach, the VEV of the respective energy-momentum tensor for spinor field has the following form

$$
\begin{aligned}
& \left\langle T_{\mu \nu}(x)\right\rangle=1 / 4 \lim _{x^{\prime} \rightarrow x} \operatorname{Tr}\left[\sigma_{\mu}\left(\nabla_{\nu}-\nabla_{\nu^{\prime}}\right)\right. \\
& \left.\quad+\sigma_{\nu}\left(\nabla_{\mu}-\nabla_{\mu^{\prime}}\right)\right] S_{F}\left(x, x^{\prime}\right) .
\end{aligned}
$$

For us it is necessary only to compute $\left\langle T_{0}^{0}(x)\right\rangle$, so we have

$$
\left\langle T_{0}^{0}(x)\right\rangle=-\frac{1}{\alpha^{2}} \lim _{x^{\prime} \rightarrow x} \operatorname{Tr} \partial_{\tau}^{2} G_{E}\left(x, x^{\prime}\right)
$$

The above limit is divergent, so in order to obtain a well defined result we should renormalize it subtracting from the Euclidean Green function, $G_{E}$, the Hadamard one, which for this case presents structure similar to the scalar case. After a long calculation we found: $B_{0}^{0}=-\frac{1-\alpha^{4}}{60}$ and $A_{0}^{0}$ a complicated integral; however we write down this latter component for specific values of the parameter $\alpha$.

1) For large solid angle deficit $(\alpha<<1)$,

$$
A_{0}^{0} \sim-\frac{1}{60} \ln \alpha+C_{0}, C_{0}=0.0104 .
$$

2) For small solid angle deficit or excess $(|\alpha-1|<<1)$,

$$
A_{0}^{0} \sim C_{1}(1-\alpha), C_{1}=0.0773 .
$$

3) For large solid angle excess $(\alpha>>1)$,

$$
A_{0}^{0} \sim-C_{3} \alpha^{4}, C_{3}=0.0173 .
$$

\section{Thermal Effects}

Now we would like to present the effects of the nonzero temperature in the formalism of quantum field theory for a massless scalar field in the G-M spacetime. We shall develop first the calculation of the respective Euclidean thermal Green function. Our subsequent analysis concerns in the calculation of the corrections to the VEV of $\left\langle\Phi^{2}(x)\right\rangle_{\beta}$ and $\left\langle T_{\mu \nu}(x)\right\rangle_{\beta}$ due to the nonzero temperature[13].

\section{A. The Euclidean Thermal Green Function}

The thermal Green function, $G_{\beta}\left(x, x^{\prime}\right)$, for a massive scalar field, non-minimally coupled with the geometry of the background spacetime, satisfies the following conditions:

(i) It obeys the differential equation

$$
\left(\square-m^{2}-\xi R\right) G_{\beta}\left(x, x^{\prime}\right)=-\frac{\delta^{(4)}\left(x, x^{\prime}\right)}{\sqrt{-g}}
$$

and

(ii) is periodic in the Euclidean time $\tau$, with period $\beta$ given by

$$
\beta=\frac{1}{\kappa_{B} T},
$$

where $\kappa_{B}$ is the Boltzman constant and $T$ the absolute temperature. 
Because the Euclidean version of our spacetime is an ultrastatic one ${ }^{3}$, the Euclidean thermal Green function can be obtained by the Schwinger-De Witt formalism as follows:

$$
G_{\beta}\left(x, x^{\prime}\right)=\int_{0}^{\infty} d s K_{\beta}\left(x, x^{\prime} ; s\right),
$$

where the thermal heat kernel can be factorized as

$$
K_{\beta}\left(x, x^{\prime} ; s\right)=\Theta_{3}\left(\frac{-i \beta \Delta \tau}{4 s} \mid \frac{i \beta^{2}}{4 \pi s}\right) K_{\infty}\left(x, x^{\prime} ; s\right),
$$

being $\Theta_{3}$ the third theta Jacobi function

$$
\Theta_{3}(z \mid \omega)=\sum_{n=-\infty}^{\infty} \exp \left(i \pi n^{2} \omega+2 i n z\right)
$$

with $\operatorname{Im}(\omega)>0 . K_{\infty}\left(x, x^{\prime} ; s\right)$ is the zero temperature heat kernel previously defined by Eq. (53) in the analysis of the massless scalar field.

After some intermediate steps we get the Euclidean thermal Green function for a scalar massless field which reads

$$
\begin{gathered}
G_{\beta}\left(x, x^{\prime}\right)=\frac{1}{8 \pi^{2} r r^{\prime}} \sum_{n=-\infty}^{\infty} \sum_{l=0}^{\infty}(2 l+1) P_{l}(\cos \gamma) \\
\times \quad Q_{\nu_{l}-1 / 2}\left(\frac{\alpha^{2}(\Delta \tau-n \beta)^{2}+r^{2}+r^{\prime 2}}{2 r r^{\prime}}\right),
\end{gathered}
$$

where we see that this Green function is a sum of a zero temperature one plus some thermal correction: $G_{\beta}\left(x, x^{\prime}\right)=G_{\infty}\left(x, x^{\prime}\right)+\bar{G}_{\beta}\left(x, x^{\prime}\right)$.

Now we are in position to calculate the thermal correction to the $\mathrm{VEV}$ of $\left\langle\Phi^{2}(x)\right\rangle_{\beta}$ and $\left\langle T_{\mu \nu}(x)\right\rangle_{\beta}$.

\section{B. The Thermal Average $\left\langle\Phi^{2}(x)\right\rangle_{\beta}$}

The singular contribution for $\left\langle\Phi^{2}(x)\right\rangle_{\beta}$ comes from the zero-temperature term in the Euclidean thermal Green function. So the procedure to renormalize this thermal average is identical to the previous one defined in Sec. 4.A.1:

$$
\begin{aligned}
\left\langle\Phi^{2}(x)\right\rangle_{\beta, \text { Ren }} & =\lim _{x^{\prime} \rightarrow x}\left[G_{\beta}\left(x, x^{\prime}\right)-G_{H}\left(x, x^{\prime}\right)\right] \\
& =\lim _{x^{\prime} \rightarrow x}\left[G_{\infty}\left(x, x^{\prime}\right)+\bar{G}_{\beta}\left(x, x^{\prime}\right)-G_{H}\left(x, x^{\prime}\right)\right] \\
& =\left\langle\Phi^{2}(x)\right\rangle_{\infty, \operatorname{Ren}} \\
& +\frac{1}{4 \pi^{2} r^{2}} \sum_{n=1}^{\infty} \sum_{l \geq 0}(2 l+1) Q_{\nu_{l}-1 / 2}\left(z_{n}^{\alpha}\right),
\end{aligned}
$$

where $z_{n}^{\alpha}=1+\frac{\alpha^{2} n^{2} \beta^{2}}{2 r^{2}}$. Because the first term in the r.h.s. of the above equation has already been discussed let us concentrate on the thermal correction. Also, here it is necessary to calculate this correction expanding a series in powers of the parameter $\Lambda$. After some intermediate steps we get the following expression:

$$
\begin{aligned}
\left\langle\bar{\Phi}^{2}(x)\right\rangle_{\beta} & =\frac{1}{12 \beta^{2}}(1+\Lambda)-\Lambda \frac{\xi \sqrt{2}}{8 \pi^{2} r^{2}} S_{1}(\beta / r) \\
& +\Lambda \frac{1}{32 \pi^{2} r^{2}} S_{2}(\beta / r)
\end{aligned}
$$

where

$$
S_{1}(\beta / r)=\sum_{n \geq 1} \int_{\rho_{n}}^{\infty} d t \frac{1}{\sqrt{\cosh t-\cosh \rho_{n}}} \frac{t}{\sinh (t / 2)}
$$

and

$$
S_{2}(\beta / r)=\sum_{n \geq 1} \int_{\rho_{n}}^{\infty} d t \frac{1}{\sqrt{\cosh t-\cosh \rho_{n}}} \frac{t}{\sinh ^{3}(t / 2)}
$$

where $\rho_{n}=\operatorname{arcosh}\left(1+\frac{\mathrm{n}^{2} \beta^{2}}{2 \mathrm{r}^{2}}\right)$.

Unfortunately it is not possible to obtain explicit results for both integrals above in terms of any set of elementary functions. So, if we want to get some concrete information about the behavior of the thermal average of $\left\langle\Phi^{2}(x)\right\rangle$ we should proceed a numerical evaluation for $S_{1}$ and $S_{2}$ for some interval of the variable $\bar{\omega}:=\beta / r$. Specifically we are interested to know this behavior in the high temperature, or great distance region, i. e., when $\bar{\omega}<<1$. By our numerical analyses[13]

\footnotetext{
${ }^{3}$ An ultrastatic spacetime admits a globally defined coordinate system in which the components of the metric tensor are timeindependent and the conditions $g_{00}=1$ and $g_{o i}=0$ hold
} 
we found the following dependences for both integrals above when $\bar{\omega}$ belong to the interval $[0.01,0.1]$ :

$$
S_{1}=\frac{c_{1}}{\bar{\omega}^{a_{1}}}
$$

and

$$
S_{2}=\frac{c_{2}}{\bar{\omega}^{a_{2}}}
$$

where $c_{1}=14.26$ with $a_{1}=0.92 \pm 0.07$ and $c_{2}=17.82$ with $a_{2}=2.02 \pm 0.02$. From these results we can infer the approximate behavior for $\left\langle\Phi^{2}(x)\right\rangle_{\beta}$ in the high temperature limit:

$$
\left\langle\bar{\Phi}^{2}(x)\right\rangle_{\beta, \text { Ren }}=\frac{1}{12 \beta^{2}}(1+1.68 \Lambda)-0.25 \frac{\Lambda \xi}{\beta r} .
$$

We can see that the leading term is independent on the non-minimal coupling $\xi$ and also on the distance from the point to the global monopole.

\section{The Thermal Average $\left\langle T_{\mu \nu}(x)\right\rangle_{\beta}$.}

The energy-momentum tensor associated to the scalar field in a general spacetime with a non-minimal coupling is given by:

$$
\begin{aligned}
T_{\mu \nu}(x) & =(1-2 \xi) \nabla_{\mu} \Phi(x) \nabla_{\nu} \Phi(x)-2 \xi\left(\nabla_{\mu} \nabla_{\nu} \Phi(x)\right) \Phi(x) \\
& +\left(2 \xi-\frac{1}{2}\right) g_{\mu \nu}(x) g^{\rho \sigma}(x) \nabla_{\rho} \Phi(x) \nabla_{\sigma} \Phi(x) \\
& -2 \xi^{2} g_{\mu \nu}(x) R(x) \Phi^{2}(x)-\xi G_{\mu \nu}(x) \Phi^{2}(x)
\end{aligned}
$$

where $G_{\mu \nu}(x)$ and $R(x)$ are, respectively, the Einstein tensor and the scalar curvature.

The thermal average of the operator energymomentum tensor can be obtained using the thermal Green function defined as usual

$$
G_{\beta}\left(x, x^{\prime}\right)=\frac{\operatorname{Tr}\left[e^{-\beta H}\left\langle T \Phi(x) \Phi\left(x^{\prime}\right)\right\rangle\right]}{T r e^{-\beta H}}
$$

So the thermal average of the energy-momentum tensor is given by

$$
\begin{aligned}
\left\langle T_{\mu \nu}(x)\right\rangle_{\beta} & =\lim _{x^{\prime} \rightarrow x}\left[(1-2 \xi) \nabla_{\mu} \nabla_{\nu^{\prime}} G_{\beta}\left(x, x^{\prime}\right)\right. \\
& -2 \xi \nabla_{\mu} \nabla_{\nu} G_{\beta}\left(x, x^{\prime}\right)-\xi G_{\mu \nu} G_{\beta}\left(x, x^{\prime}\right) \\
& +\left(2 \xi-\frac{1}{2}\right) g_{\mu \nu} g^{\rho \sigma^{\prime}} \nabla_{\rho} \nabla_{\sigma^{\prime}} G_{\beta}\left(x, x^{\prime}\right) \\
& \left.\left.-2 \xi^{2} g_{\mu \nu} R(x) G_{\beta}\left(x, x^{\prime}\right)\right)\right] .
\end{aligned}
$$

We have already mentioned that the thermal Green function associated with a scalar field in G-M spacetime can be written as a sum of a zero temperature one plus thermal corrections; so the above expression can be written as

$$
\left\langle T_{\mu \nu}(x)\right\rangle_{\beta}=\left\langle T_{\mu \nu}(x)\right\rangle_{\infty}+\left\langle\bar{T}_{\mu \nu}(x)\right\rangle_{\beta}
$$

The general structure of the independent temperature term in the r.h.s. of the above equation has already been discussed previously in Sec. 4.A.2. So, by this reason, we shall concentrate on the purely thermal correction, $\left\langle\bar{T}_{\mu \nu}(x)\right\rangle_{\beta}$. For the sake of simplicity we shall analyze its zero-zero component only:

$$
\begin{aligned}
\left\langle\bar{T}_{00}\right\rangle_{\beta} & =\lim _{x^{\prime} \rightarrow x}\left[-\partial_{\tau}^{2} \bar{G}_{\beta}\left(x, x^{\prime}\right)\right. \\
& +\left(2 \xi-\frac{1}{2}\right) g^{\rho \sigma^{\prime}}\left(x, x^{\prime}\right) \nabla_{\rho} \nabla_{\sigma^{\prime}} \bar{G}_{\beta}\left(x, x^{\prime}\right) \\
& \left.+\frac{1}{2} \xi(1-4 \xi) R(x) \bar{G}_{\beta}\left(x, x^{\prime}\right)\right]
\end{aligned}
$$

The development of this equation is a long one. Our final result is

$$
\begin{aligned}
\left\langle\bar{T}_{00}\right\rangle_{\beta} & =\frac{\alpha^{2}}{4 \pi^{2} r^{4}} \sum_{n \geq 1} \sum_{l \geq 0}(2 l+1)\left[\Xi_{n} Q_{\nu_{l}-1 / 2}^{(2)}\left(z_{n}\right)\right. \\
& \left.+\Theta_{n} Q_{\nu_{l}-1 / 2}^{(1)}\left(z_{n}\right)+\Psi_{l} Q_{\nu_{l}-1 / 2}\left(z_{n}\right)\right]
\end{aligned}
$$

where $Q_{\nu}^{(k)}(z)$ are the associated Legendre functions, $z_{n}=1+\frac{n^{2} \alpha^{2}}{2} \bar{\omega}^{2}$ and 


$$
\begin{aligned}
\Xi_{n} & =\frac{1}{\alpha^{2} n^{2} \bar{\omega}^{2}+4}\left[2 \xi\left(n^{2} \bar{\omega}^{2}-4\right)-1 / 2\left(n^{2} \bar{\omega}^{2}+4\right)\right] \\
\Theta_{n} & =\frac{1}{n \bar{\omega} \alpha^{3} \sqrt{n^{2} \bar{\omega}^{2}+4}}\left[2 \xi\left[\left(3 n^{2} \bar{\omega}^{2}-2\right) \alpha^{2}-2\right]\right. \\
& \left.-1 / 2\left[\left(3 n^{2} \bar{\omega}^{2}+2\right) \alpha^{2}-2\right]\right] \\
\Psi_{l} & =(2 \xi-1 / 2)+\frac{l(l+1)}{\alpha^{2}}+\frac{\xi}{2}(1-4 \xi) \frac{\alpha^{2}-1}{\alpha^{2}} .
\end{aligned}
$$

Also in this case it is not possible to obtain some concrete information about the behavior of $\left\langle T_{00}(x)\right\rangle_{\beta}$ with the temperature without to adopt the following procedures: (i) To expanding a series in powers of the parameter $\Lambda=1-\alpha^{2}$, and (ii) to proceed the summation in the angular quantum number $l$. After doing that, some expressions obtained allow us to proceed the summation in $n$. Unfortunately we also could not develop all the integrals that appears and consequently the summations in $n$; again, here we had to develop some numerical evaluations for these terms in the region where $\bar{\omega}<<1$. Our final result is:

$$
\begin{aligned}
\left\langle\bar{T}_{00}(x)\right\rangle_{\beta} & =-\frac{\pi^{2}}{30 \beta^{4}}+\left(\frac{1}{24}-\frac{\xi}{6}\right) \frac{1}{r^{2} \beta^{2}}+\left[\Omega_{1}(\xi) \frac{1}{\beta^{4}}\right. \\
& \left.+\Omega_{2}(\xi) \frac{1}{r^{2} \beta^{2}}+\frac{1}{4 \sqrt{2} \pi^{2} r^{4}} \bar{F}(\bar{\omega})\right] \Lambda,(120)
\end{aligned}
$$

where

$$
\Omega_{1}(\xi)=-\frac{\pi^{2}}{180}(11+52 \xi)
$$

and

$$
\Omega_{2}(\xi)=-\frac{1}{768}\left(64 \xi^{2}+1725 \xi-832\right) .
$$

The function $\bar{F}(\bar{\omega})$ contains all the integrals which were not possible to be expressed in terms of elementary functions and consequently to proceed the summations in $n$. Analyzing all the terms in $\bar{F}(\bar{\omega})$ numerically, in the high temperature regime we have obtained that the leading term are, approximately proportional to $1 / \bar{\omega}^{4}$, followed by terms proportional to $1 / \bar{\omega}^{3}$ and $1 / \bar{\omega}^{2}$.

As our final conclusion about the thermal effects in the renormalized VEV of the operators $\Phi^{2}(x)$ and $T_{00}(x)$ we can say that in the high temperature regime we have:

$$
i)\left\langle\Phi^{2}(x)\right\rangle_{\text {Ren }}^{T}=\left\langle\Phi^{2}(x)\right\rangle_{\text {Ren }}^{0}+T^{2} f(\gamma)
$$

and

$$
i i)\left\langle T_{00}(x)\right\rangle_{\text {Ren }}^{T}=\left\langle T_{00}(x)\right\rangle_{\text {Ren }}^{0}+T^{4} g(\gamma)
$$

where $f$ and $g$ are functions of the dimensionless parameter $\gamma=\kappa_{B} r T$. The analytic form for these functions were obtained by us, up the first order in $\Lambda$.

\section{Other Applications}

\section{A. Topological Inflation}

Topological defects can be seeds for inflation[15]. Vilenkin and Linde claimed that topological defects expand exponentially if $\eta_{0}>O\left(m_{P}\right)$.

In the particular case of a global monopole, $\alpha^{2}=$ $1-8 \pi G \eta_{0}^{2}$ becomes negative and the solid angle deficit ( $\left.\delta \Omega=4 \pi\left(8 \pi G \eta_{0}^{2}\right)\right)$ bigger than $4 \pi$. The role of the radial and time coordinates interchanges. The exterior solution corresponds to the G-M solution is no longer static.

\section{B. Gravitating Magnetic Monopole}

The occurrence of a deficit angle is a consequence of the nontrivial topology of the bosonic field configuration outside the the monopole. The non vanishing gradients along nonradial directions imply an energy density that falls off slowly:

$$
T_{00} \sim \phi_{, i}^{a} \phi_{, i}^{a} \sim \frac{\eta^{2}}{r^{2}} .
$$

(This result also points out that the total Newtonian mass contained within $r$ is $M(r) \sim 4 \pi G \eta_{0}^{2} r$. If we take the radial extent of a Galaxy to be $R_{G} \simeq 15 \mathrm{Kpc}$ and consider a typical grand unified theory with $\eta_{0} \simeq$ $10^{16} \mathrm{Gev}$, this mass turns out to be of order $10^{69} \mathrm{Gev}$, which is ten times the total mass of a typical Galaxy.)

However considering a gravitating magnetic monopole, the gauge covariant derivative of the scalar filed vanishes outside the monopole:

$$
\left(D_{i} \phi\right)^{a}=\partial_{i} \phi^{a}-e \epsilon_{a b c} A_{i}^{b} \phi^{c} r \longrightarrow 0 .
$$


So there is no deficit angle.

\section{Condensed Matter}

The effective gravity arise in many condensed matter system. The typical examples are the crystals with dislocations and disclinations linear defects. However it appears that the superfluid ${ }^{3} \mathrm{He}-A$ provides the most adequate analogies for relativistic models of the effective gravity.

The quasiparticles in ${ }^{3} \mathrm{He}-\mathrm{A}$ are chiral and massless fermions. Under specific circumstances these fermions are 'relativistic' with the spectrum[16]

$$
E^{2}(k)+g^{i j}\left(k_{i}-e A_{i}\right)\left(k_{j}-e A_{j}\right)=0 .
$$

Here $\vec{A}$ is the dynamical vector potential of the induced electromagnetic field, $\vec{A}=k_{F} \hat{l}$ and $\hat{l}$ is the unity vector in the moment space.

The metric tensor of the effective space which governs the motion of the fermions is given by

$$
g^{i j}=-c_{\perp}^{2}\left(\delta^{i j}-l^{i} l^{j}\right)-c_{\|}^{2} l^{i} l^{j}, g^{00}=1 .
$$

Here $c_{\perp}$ and $c_{\|}=v_{F}\left(c_{\perp}<<c_{\|}\right)$are the speed of the light propagating transverse to $\hat{l}$ and along $\hat{l}$, respectively.

For the specific case where $\hat{l}=\hat{r}$, we get

$$
d s^{2}=d t^{2}-\frac{d r^{2}}{a^{2}}-r^{2} d \Omega^{2},
$$

where $a^{2}=c_{\|}^{2} / c_{\perp}^{2}>1$. In this case we have a metric spacetime analogous to the G-M one with a solid angle excess.

\section{Concluding Remarks}

In the present paper we have reviewed some of the most important aspects about the global monopole spacetime and its consequences on the the classical and quantum movement of a charged particle. Also we have calculated the vacuum polarization effects due to massless scalar and fermionic fields in this manifold, and the effects of the nonzero temperature on these quantities.

Besides the main results exhibited in this paper as mentioned above, the formalisms developed here can be applied to other sub-areas of physics since the inflation problem in cosmology to condensed matter system. For all these reasons we believe that the physics in the global monople spacetime is very rich and deserves to be studied.

\section{Acknowledgment}

My acknowledgments to all my collaborators (see references $([6]),([7]),([12])$ and $([13]))$ who made this work possible. This work was partially supported by $\mathrm{CNPq}$.

\section{References}

[1] T. W. B. Kibble, J. Phys. A 9, 1387 (1976). A. Vilenkin, Phys. Rep. 121, 263 (1985).

[2] M. Bariola and A. Vilenkin, Phys. Rev. Lett. 63, 341 (1989).

[3] C. O. Lousto and N. Sanchez, Phys. Lett. B212, 411 (1988).

[4] D. Harari and C. O. Lousto, Phys. Rev. D 42, 2626 (1990).

[5] A. G. Smith, in Proceeding of the Symposium on the Formation and Evolution of Cosmic String, ed. by G. W. Gibbons, S. W. Hawking and T. Vaschapati, Cambridge Univ. Press, Cambridgem, England, 1990.

[6] E. R. Bezerra de Mello and C. Furtado, Phys. Rev. D 56, 1345 (1997).

[7] A. A. Rodrigues Sobreira and E. R. Bezerra de Mello, Grav. and Cosmology 5, 177 (1999).

[8] W. A. Hiscock, Class. Quantum Grav. 7, L 235 (1990).

[9] F. D. Mazzitelli and C. O. Lousto, Phys. Rev. D 43, 468 (1991).

[10] N. D. Birrell and P. C. W. Davies, Quantum Field in Curved Space (Cambridge University Press, Cambridge, England, 1982).

[11] J. D. Bjorken and S. Drell, Relativistic Quantum Mechanics (McGraw-Hill, New York, 1964).

[12] E. R. Bezerra de Mello, V. B. Bezerra and N. R. Khusnutdinov, Pys. Rev. D 60, 063506 (1999).

[13] F. C. Cabral and E. R. Bezerra de Mello. Class. Quantum Grav. 18, 1637 (2001).

[14] M. Abramowitz and I. A. Stegum, Handbook of Mathematical Functions (Dover, New York, 1972).

[15] A. L. Vilenkin, Phys. Rev. Lett. 72, 3137 (1994). A. D. Linde, Phys. Lett. B 327, 208 (1994).

[16] G. E. Volovik, JETP Lett. 67, 698 (1998). 